\title{
Wählen im Blick Europas. Die Beobachtung der Bundestagswahl 2009 durch die OSZE
}

\author{
Hans-Jörg Schmedes
}

„Das gab es noch nie“, vermeldete die Internetausgabe des Spiegels ${ }^{1}$ einleitend in einem Artikel unmittelbar nach Bekanntwerden der Nachricht am Nachmittag des 9. August 2009 mit gewohntem Unterton, „,[d]ie europäische Organisation für Sicherheit und Zusammenarbeit wird zum ersten Mal Wahlbeobachter nach Deutschland entsenden - um den korrekten Ablauf der Bundestagswahl zu prüfen“. Was in dem Artikel zunächst in unmittelbaren Zusammenhang mit der kurz zuvor verweigerten Zulassung einiger Kleinparteien zum Urnengang durch den Bundeswahlausschuss gestellt wurde, entpuppte sich in der parallelen Berichterstattung anderer Medien als wenig spektakuläre und vor allem nicht ungewöhnliche Entscheidung der Organisation für Sicherheit und Zusammenarbeit in Europa (OSZE) ohne konkreten Anlass für Zweifel an der Integrität des Prozesses. Vielmehr wollten sich die internationalen Inspektoren „vor allem die Abläufe vor und während der Wahlen anschauen und Vorschläge für Verbesserungen erarbeiten "2; der OSZE-Sprecher Jens-Hagen Eschenbächer beklagte laut Süddeutscher Zeitung vor diesem Hintergrund auch die „sehr irreführenden Interpretationen “ in Deutschland. ${ }^{3}$

Dennoch wurde die Entscheidung der OSZE für die Beobachtung der Bundestagswahl vielfach mit Überraschung zur Kenntnis genommen, denn bislang brachte man die internationale Beobachtung von Wahlen entweder mit Staaten in Transformationsprozessen hin zu einer demokratischen Regierungsform oder mit etablierten Demokratien in Verbindung, deren vorangegangene Wahlen von vermeintlichen oder erwiesenen Unregelmäßigkeiten gekennzeichnet waren, wie beispielsweise die US-amerikanischen Präsidentschaftswahlen im November 2000. Beides trifft auf Deutschland jedoch nicht zu. Vielmehr ist das Vertrauen der Öffentlichkeit in die allgemeine Integrität des Wahlprozesses hierzulande als sehr hoch einzuschätzen, wie die OSZE selbst als ein Ergebnis ihrer Bedarfsermittlungsmission im Vorfeld der Entscheidung zugunsten der Beobachtung in Deutschland hervorhob. ${ }^{4} \mathrm{Um}$

* Der Autor ist Wissenschaftlicher Mitarbeiter des Bundestagsabgeordneten Peter Friedrich und hat an Wahlbeobachtungsmissionen der OSZE und der EU in Georgien (2004), in der Ukraine (2004, 2010), in Mazedonien (2005), in Uganda (2006) und in Kenia (2007) teilgenommen. Der Beitrag gibt ausschließlich seine persönliche Auffassung wieder.

1 Spiegel Online, Parteien-Zulassung: OSZE schickt Wahlbeobachter nach Deutschland, 9. August 2009, http://www.spiegel.de/politik/deutschland/0,1518,641353,00.html (Abruf am 20. August 2009).

2 Focus Online, Wahlen: OSZE-Wahlbeobachter schauen auf Bundestagswahl, 9. August 2009, http://www.focus.de/politik/deutschland/wahlen-osze-wahlbeobachter-schauen-auf-bundestagswahl_aid_424651.html (Abruf am 20. August 2009).

3 Daniel Brössler, OSZE auf West-Mission. Internationale Beobachter sollen Bundestagswahl überwachen, um Kritik an „doppelten Standards“ zu entkräften, in: SZ vom 11. August 2009, S. 5.

4 Vgl. Organization for Security and Co-operation in Europe / Office for Democratic Institutions and Human Rights (OSCE / ODIHR), Federal Republic of Germany. Elections to the Federal Parliament (Bundestag), OSCE / ODIHR Needs Assessment Mission Report, http://www.osce. org/item/39164.html (Abruf am 21. August 2009), S. 2, S. 10. 
zu verstehen, warum der Urnengang dennoch von einer internationalen Organisation beobachtet wurde, müssen einige Fragen geklärt werden:

Was genau verbirgt sich hinter einer Wahlbeobachtung, und welche Einrichtungen engagieren sich in diesem Bereich? Wer trifft die Entscheidung über die Entsendung einer Beobachtungsmission, und wer bewertet die Beobachtungsergebnisse? Auf dieser Basis wird nachvollziehbar, wie es dazu kam, dass die Bundestagswahlen 2009 von der OSZE beobachtet worden sind, ohne dass der Ablauf vorangegangener Wahlen Zweifel an einem freien und fairen Ablauf des Urnenganges begründet hätte, auf welche Weise die Wahlen in Deutschland beobachtet wurden und zu welchen Ergebnissen die OSZE-Experten gelangten.

\section{Die Beobachtung von Wahlen als internationales Demokratisierungsinstrument}

Die internationale Beobachtung von Wahlen kann auf eine langjährige Geschichte zurückblicken, wenngleich sie sich erst infolge der demokratischen Umbrüche in den Ländern Mittel-, Ost- und Südosteuropas nach dem Fall der Berliner Mauer als systematisches Mittel der Demokratisierungshilfe herausgebildet hat. 1857 wurden mit den Wahlen in den Fürstentümern Moldau und Walachei erstmals Urnengänge unter internationaler Ägide beobachtet, wenngleich damals zwischen verfeindeten Staaten. Dem folgte die Beobachtung zahlreicher Plebiszite über Grenzverschiebungen infolge des Ersten Weltkrieges. In einer zweiten Phase dienten internationale Wahlbeobachtungsmissionen als Hilfsmittel der Entkolonialisierung, auf das die Vereinten Nationen (UN) bei zahlreichen Abstimmungen in Afrika und Asien in den 1950er und 1960er Jahren zurückgriffen. Mit der Beobachtung der Wahlen in Nicaragua im Februar 1990 durch die UN trat erstmals die Bedeutung des Instruments bei der Beendigung innerstaatlicher Auseinandersetzungen hervor, ehe die Wichtigkeit von Wahlbeobachtungen bei der Etablierung demokratischer Strukturen innerhalb von staatlichen Transformationsprozessen seit Ende des Kalten Krieges stetig zunahm. ${ }^{5}$

Die Generalversammlung der UN hat mit Resolution 46/137 vom 17. Dezember 1991 für Maßnahmen zur Hilfe bei Wahlen eine organisatorische Grundlage und einen hochrangigen Ansprechpartner geschaffen sowie mit der Electoral Assistance Unit eine Stelle eingerichtet, die unterschiedliche Formen der Wahlunterstützung anbietet. Mittlerweile firmiert diese unter der Bezeichnung Electoral Assistance Division und ist im Department of Political Affairs angesiedelt. Die UN kann nur auf Bitten des betreffenden Mitgliedstaates tätig werden und diesem Hilfe bei der Organisation und Durchführung, aber auch bei der Überwachung und Überprüfung von Wahlen leisten. ${ }^{6}$ Seit den frühen 1990 er Jahren etablierten

5 Vgl. Eric C. Bjornlund, Beyond Free and Fair. Monitoring Elections and Building Democracy, Washington D.C. 2004, S. 53 - 74; Ekkehard Münzing, Internationale Wahlbeobachtung nach dem Ende des Ost-West-Konflikts, Berlin / München 2005, S. 126 - 152; Judith Kelley, Assessing the Complex Evolution of Norms: The Rise of International Election Monitoring, in: International Organization, 62. Jg. (2008), H. 2, S. 221 - 255; Stefan Mair, Internationale Präsenz und nationale Souveränität. Wahlbeobachtung: ein expandierendes Betätigungsfeld der Staatengemeinschaft, in: Vereinte Nationen, 42. Jg. (1994), H. 4, S. 133 - 140, S. 133 f.

6 Vgl. Robin Ludwig, The UN's Electoral Assistance: Challenges, Accomplishments, Prospects, in: Edward Newman / Roland Rich (Hrsg.), The UN Role in Promoting Democracy: Between Ideals and Reality, Tokyo / New York / Paris 2004, S. 169 - 187; Stefan Mair, a.a.O. (Fn. 5), S. 133 f. 
sich im Bereich der Beobachtung von Wahlen zahlreiche weitere internationale sowie nichtstaatliche Organisationen, darunter in besonderem Maße die OSZE, aber auch die Europäische Union (EU), die Organisation of American States (OAS), das National Democratic Institute of the United States (NDI), das International Republican Institute (IRI), das International Institute for Democracy and Electoral Assistance (IDEA), die International Foundation for Election Systems (IFES), das Carter Center, der Europarat, die Afrikanische Union (AU), die Gemeinschaft Unabhängiger Staaten (GUS) sowie der Commonwealth. ${ }^{7}$

Das Instrument der Wahlbeobachtung kann die Transparenz von Wahlen und dadurch das öffentliche Vertrauen in den Wahlprozess sowie die Legitimität und Stabilität der daraus hervorgehenden Regierung gerade in jungen Demokratien stärken. Die Anwesenheit einer internationalen Beobachtermission kann eine Abschreckung gegen Wahlbetrug darstellen und durch die Veröffentlichung ihrer Beobachtungsergebnisse Verfahrensmängel zu verbessern helfen. ${ }^{8}$ Im Falle irregulärer oder gefälschter Wahlen kann eine Beobachtermission sowohl für die internationale Staatengemeinschaft als auch für die Öffentlichkeit des betroffenen Landes den Beleg für das Ausmaß der Unregelmäßigkeiten liefern und nationaler Opposition wie internationalen Gremien dadurch die Grundlage zur Nichtanerkennung amtlich verkündeter Wahlergebnisse liefern. Hieraus kann entscheidender Einfluss auf den weiteren Verlauf des Geschehens erwachsen, wie dies beispielsweise bei den „bunten Revolutionen" im November 2003 in Georgien, im November 2004 in der Ukraine und im März 2005 in Kirgisien sowie bei den blutigen Unruhen in Kenia im Nachgang der Präsidentschaftswahlen am 27. Dezember 2007 geschehen ist. Beobachtungsmissionen der OSZE beziehungsweise der EU haben in diesen Fällen das Ausmaß systematischer Unregelmäßigkeiten im Wahlverlauf durch ihre Arbeit belegen können und dadurch einen grundlegenden Beitrag für einen politischen Wandel infolge der gefälschten Wahlen geleistet. ${ }^{9}$ Entscheidend für eine solche Rolle ist die Glaubwürdigkeit einer Mission und ihrer Beobachtungsergebnisse, für die wiederum ein möglichst hohes Maß an Objektivität in der Beobachtung und an Unabhängigkeit in der Bewertung Voraussetzung ist. ${ }^{10}$

7 Vgl. beispielsweise Eric C. Bjornlund, a.a.O. (Fn. 5), S. 54; Ekkehard Münzing, a.a.O. (Fn. 5), S. 155 - 264; sowie Rachel Ricker, Two (or Five, or Ten) Heads are Better than One: The Need for an Integrated Effort to International Election Monitoring, in: Vanderbilt Journal of Transnational Law, 39. Jg. (2006), H. 4, S. 1373 - 1400, S. $1378-1387$.

8 Vgl. Peter Eicher, Improving OSCE Election Observation, in: Security and Human Rights, 20. Jg. (2009), H. 4, S. $264-276$, S. $264-266$.

9 Vgl. Rick Fawn, Battle Over the Box: International Election Observation Missions, Political Competition and Retrenchment in the Post-Soviet Space, in: International Affairs, 82. Jg. (2006), H. 6, S. 1133 - 1153, S. 1139 - 1141; Geert-Hinrich Ahrens, Die Präsidentschaftswahlen in der Ukraine. Die schwierige Mission der OSZE / ODIHR-Wahlbeobachter (August 2004 bis Januar 2005), in: Zentrum für Europäische Integrationsforschung (ZEI), Discussion Paper C 151, Bonn 2005, http://www.zei.de/download/zei_dp/dp_c151_ahrens.pdf (Abruf am 21. August 2009); Ted Dagne, Kenya: The December 2007 Elections and the Challenges Ahead, CRS Report for Congress, Washington D.C. 2008, http://www.dtic.mil/cgi-bin/GetTRDoc?AD=ADA487565\& Location=U2\&doc=GetTRDoc.pdf (Abruf am 20. Januar 2010); Jacqueline Klopp / Prisca Kamungi, Violence and Elections: Will Kenya Collapse?, in: World Policy Journal, 24. Jg. (2004), H. 4, S. $11-18$, S. 11.

10 In einer jüngst erschienenen vergleichenden Analyse argumentiert Judith Kelley (dies., D-Minus Elections: The Politics and Norms of International Election Observation, in: International Organization, 63. Jg. (2009), H. 4, S. 765 - 787, S. 766) allerdings auf Basis der Daten von knapp 600 Wahlbeobachtungsmissionen, dass Objektivität und Unabhängigkeit nicht einfach zu errei- 
Deutschland beteiligt sich im Rahmen seiner Mitgliedschaften an Beobachtungen der EU und der OSZE; deutsche Abgeordnete nehmen zudem an Missionen der Parlamentarischen Versammlungen von OSZE, Europarat und NATO sowie des Europäischen Parlaments teil. ${ }^{11}$ Mit Amtsantritt der rot-grünen Bundesregierung 1998 wurde die Demokratisierungshilfe zu einem „neuen Schwerpunkt in der deutschen Außenpolitik“12. Sie erfolge, wie ein im Mai 2004 vorgestellter Aktionsplan der Bundesregierung unterstreicht, „in erster Linie durch Wahlhilfe und internationale Wahlbeobachtung“ und stelle einen „Beitrag zur Konsolidierung und Stärkung junger Demokratien und Teil einer konfliktvorbeugenden Diplomatie"13 dar. Dem Instrument der internationalen Wahlbeobachtung misst die Bundesregierung in mehrfacher Hinsicht Bedeutung zu, wie sie an anderer Stelle ${ }^{14}$ hervorhebt: Neben einer Verbesserung der „Bedingungen zur Durchführung von freien und fairen Wahlen und somit [.. der] Voraussetzungen für das Vertrauen in den Demokratisierungsprozess“ solle die Beobachtung von Wahlen „dazu beitragen, Manipulationen vor, während und nach einer Wahl zu verhindern, indem sie deren Vorbereitung und Durchführung verfolgt und kritisch bewertet“. Zudem werde durch das Instrument „das Interesse der internationalen Gemeinschaft an der demokratischen Entwicklung eines Landes“ verdeutlicht, was die bürgerlichen und politischen Menschenrechte innerhalb des Landes zu fördern helfe. Die gesteigerte Bedeutung von Fragen der zivilen internationalen Krisenprävention, Konfliktlösung und Friedenskonsolidierung sowie von internationalen Wahlbeobachtungen fand ihren Ausdruck auch in der Gründung des Zentrums für Internationale Friedenseinsätze (ZIF) im Juni 2002. Der Einrichtung, deren Gesellschafter die Bundesrepublik Deutschland ist und die sich aus Mitteln des Auswärtigen Amts finanziert, obliegt die Auswahl, Ausbildung und Entsendung deutschen Personals in zivilen Friedenseinsätzen, darunter auch Wahlbeobachtungsmissionen der OSZE und der EU. ${ }^{15}$

\section{Standards demokratischer Wahlen und ihrer Beobachtung}

Auf internationaler Ebene existieren weltweit zahlreiche Standards für demokratische Wahlen. Ausgehend von der „Allgemeinen Erklärung der Menschenrechte“ durch die UN am 10. Dezember 1948, die in Artikel 21 Absatz 3 den „Willen des Volkes“ als „Grundlage für

chen sind, sondern Wahlbeobachter in ihren Erwägungen bewusst oder unbewusst von einer Vielzahl von Normen und Interessenlagen beeinflusst werden können: „[E]lection monitors sometimes endorse elections to protect the interests of their member states or donors or to accommodate other compelling but tangential norms."

11 Vgl. Auswärtiges Amt, Demokratisierungshilfe / Wahlbeobachtung, http://www.auswaertigesamt.de/diplo/de/Aussenpolitik/Themen/Krisenpraevention/Massnahmen/Wahlbeobachtungen. html (Abruf am 31. Januar 2010).

12 Bundesregierung, Aktionsplan „Zivile Krisenprävention, Konfliktlösung und Friedenskonsolidierung", Berlin 2004, S. 37, http://www.auswaertiges-amt.de/diplo/de/Aussenpolitik/Themen/Krisenpraevention/Downloads/Aktionsplan-De.pdf (Abruf am 3. September 2009).

13 Ebenda, S. 37.

14 Vgl. Auswärtiges Amt, a.a.O. (Fn. 11).

15 Vgl. Winrich Kühne (Hrsg.), 7 Jahre Zentrum für Internationale Friedenseinsätze (ZIF), Berlin 2009, http://www.zif-berlin.org/fileadmin/uploads/analyse/dokumente/veroeffentlichungen/7_ Jahre_ZIF_07_09.pdf (Abruf am 31. Januar 2010). 
die Autorität der öffentlichen Gewalt“ charakterisiert und den Anspruch auf „regelmäßige, unverfälschte, allgemeine und gleiche Wahlen mit geheimer Stimmabgabe oder einem gleichwertigen freien Wahlverfahren" 16 festschreibt, sowie dem "Internationalen Pakt über bürgerliche und politische Rechte“ der UN vom 16. Dezember 1966, der diese Prinzipien in konkrete Rechte gerinnen lässt, haben zahlreiche inter- und supranationale Organisationen Standards demokratischer Wahlen in unterschiedlichen Detaillierungsgraden etabliert. ${ }^{17}$ Gemeinhin wird auf das Konzept „freier und fairer Wahlen“ als gemeinsamer Standard $^{18}$ für die Bewertung der demokratischen Qualität von Wahlen rekurriert, das von Robert A. Dahl ${ }^{19}$ als eine der institutionellen Grundvoraussetzungen von Demokratie charakterisiert worden ist. Diesen Zusammenhang brachte der damalige UN-Generalsekretär Kofi Annan auf einer Konferenz in Warschau im Juni 2000 prägnant auf den Punkt: „While democracy must be more than free elections, it is also true [...] that it cannot be less. " 20

Bislang ist der Standard „freier und fairer Wahlen“ allerdings nicht durch international allgemein anerkannte Kriterien operationalisiert worden, sondern wurde und wird von internationalen Organisationen unterschiedlich ausgelegt. ${ }^{21}$ In einer demokratietheoretischen Abhandlung des Standards haben die dänischen Politikwissenschaftler Jørgen Elklit und Palle Svensson seine beiden Bestandteile definiert: Die Dimension der „Freiheit“ bezieht sich demzufolge auf die Möglichkeit der Wähler zur Teilnahme an der Wahl ohne jegliche Form des Zwangs oder einer Restriktion. „Fairness“ sei gleichzusetzen mit dem Begriff der Unvoreingenommenheit und beinhalte sowohl die vorschriftsmäßige Anwendung bestehender Regeln als auch die angemessene Verteilung relevanter Ressourcen unter den Konkurrenten. ${ }^{22}$ Für eine konkrete Anwendung bei der Beobachtung und Bewertung von Wahlen bleiben diese Definitionen allerdings zu vage.

1990 hat die OSZE, damals noch unter der Bezeichnung Konferenz für Sicherheit und Zusammenarbeit in Europa (KSZE), in ihrem vielbeachteten Kopenhagener Dokument gemeinsame Standards der Teilnehmerstaaten für demokratische Institutionen und Rechtsstaatlichkeit formuliert. Als Bestandteil der menschlichen Dimension, die neben der politisch-militärischen sowie der wirtschaftlichen Dimension einen der drei Themenbereiche

16 United Nations, Office of the High Commissioner of Human Rights, Universal Declaration of Human Rights, http://www.ohchr.org/EN/UDHR/Pages/Language.aspx?LangID=ger (Abruf am 31. Januar 2010).

17 Vgl. Victor-Yves Ghebali, Die Debatte über Wahlstandards und Standards der Wahlbeobachtung in der OSZE: zwischen technischer Notwendigkeit und Politisierung, in: Institut für Friedensforschung und Sicherheitspolitik an der Universität Hamburg (Hrsg.), OSZE-Jahrbuch 2005, Baden-Baden 2006, S. 241 - 257. Eine gute Zusammenstellung über die internationalen Standards unterschiedlicher Organisationen findet sich in dem von der Europäischen Kommission gemeinsam mit dem „Network of Europeans for Electoral and Democracy Support" herausgegebenen „Compendium of International Standards for Elections“, London / Brüssel 2007, http://www. needs-network.org/pdfs/Needs_Comp_2007_(web).pdf (Abruf am 28. Oktober 2009).

18 Vgl. Eric C. Bjornlund, a.a.O. (Fn. 5), S. $96-121$.

19 Robert A. Dahl, Democracy and Its Critics, New Haven / London 1989, S. 221 f.

20 UN Secretary General Kofi Annan's Closing Remarks to the Ministerial, http://www.demcoalition.org/pdf/un_secertary_gen_kofi_annan.pdf (Abruf 9. Januar 2010), S. 3.

21 Vgl. Eric C. Bjornlund, a.a.O. (Fn. 5), S. 94 - 128; Rachel Ricker, a.a.O. (Fn. 7).

22 Vgl. Jorgen Elklit / Palle Svensson, The Rise of Election Monitoring: What Makes Elections Free and Fair?, in: Journal of Democracy, 8. Jg. (1997), H. 3, S. 32 - 46, S. 35. 
der Organisation darstellt ${ }^{23}$, gehören diese Standards zu einem Komplex, der in verschiedenen KSZE-Dokumenten explizit als eine „nicht ausschließlich innere Angelegenheit der Nationalstaaten " 24 beschrieben wird.

Im Kopenhagener Dokument vom 29. Juni 1990 bekräftigen die Teilnehmerstaaten in $\$$ 6 , „daß der durch regelmäßige und unverfälschte Wahlen frei und gerecht zum Ausdruck gebrachte Wille des Volkes die Grundlage für die Autorität und Rechtmäßigkeit jeder Regierung bildet“ ${ }^{“ 25}$. $\$ 7$ konkretisiert diese Prinzipien um zentrale Anforderungen, die mit den sieben Schlüsselwörtern „Universalität“, „Gleichheit“, „Gerechtigkeit“, „Geheimhaltung“, „Freiheit“, „Transparenz“ und „Nachprüfbarkeit“ zusammengefasst werden können. ${ }^{26} \$ 8$ des Dokuments hebt die Vorteilhaftigkeit von inländischen wie ausländischen Wahlbeobachtern hervor und verpflichtet die Teilnehmerstaaten, zur Beobachtung landesweiter Wahlen einzuladen und die Beobachtung von Wahlen auch unterhalb der nationalen Ebene zu ermöglichen. ${ }^{27}$ Zentrale Kontakt- und Koordinierungsstelle der menschlichen Dimension und somit auch der Beobachtung von Wahlen ist das mittlerweile aus dem ursprünglichen Büro für freie Wahlen hervorgegangene Büro für Demokratische Institutionen und Menschenrechte (BDIMR), das besser unter seinem englischen Akronym ODIHR (Office for Democratic Institutions and Human Rights) bekannt ist. ${ }^{28}$ Die Gesamtverantwortung für die Entscheidung über die Entsendung einer Wahlbeobachtungsmission, für deren Durchführung sowie für die Bewertung der Beobachtungsergebnisse und der daraus abgeleiteten Empfehlungen liegt beim Direktor des ODIHR. ${ }^{29}$

Mit der Verpflichtung zur beständigen Einladung an die OSZE-Teilnehmerstaaten zur gegenseitigen Beobachtung von Wahlen in $\$ 8$ des Kopenhagener Dokuments haben souveräne Staaten das Instrument der Wahlbeobachtung zum ersten Mal auf eine institutionalisierte Basis gestellt. ${ }^{30}$ Seitdem wurden sowohl die Strukturen von Beobachtungsmissionen als auch die Normen zur Beurteilung des Ablaufs von ODIHR zunehmend systematisiert, und der Bewertung der demokratischen Qualität von Wahlen anhand der Dimensionen „Freiheit“ und „Gerechtigkeit“ wird ein zunehmend komplexeres Verständnis des Wahlprozesses zugrunde gelegt. Dieses Verständnis geht weit über den eigentlichen Urnengang hinaus, denn als mindestens genauso bedeutsam wie die Stimmabgabe und die Addierung der abgegebenen Stimmen auf lokaler, regionaler und nationaler Ebene werden die vorangehende und die sich an den Wahltag unmittelbar anschließende Phase betrachtet, in denen ebenfalls weitreichende Manipulationsmöglichkeiten existieren. Diese beginnen mit Lü-

23 Vgl. Kurt P. Tudyka, Das OSZE-Handbuch. Die Organisation für Sicherheit und Zusammenarbeit von Vancouver bis Wladiwostok, Opladen 2002, S. 109 f.; siehe auch OSCE Handbook, Wien 2007, S. 80 - 99, http://www.osce.org/item/22286.html?ch=1002 (Abruf am 30. Januar 2010).

24 Vgl. Kurt P. Tudyka, a.a.O. (Fn. 23), S. 49, S. 145.

25 Dokument des Kopenhagener Treffens der Konferenz über die Menschliche Dimension der KSZE („Kopenhagener Dokument“), S. 7, Kopenhagen 1990, http://www.osce.org/item/13992. html?lc=DE (Abruf am 5. November 2009).

26 Vgl. ebenda, S. 7 f.; Victor-Yves Ghebali, a.a.O. (Fn. 17), S. 243 f.; OSCE / ODIHR, Election Observation. A Decade of Monitoring Elections: The People and the Practice, Warschau 2008, S. 29, http://www.osce.org/item/17148.html?ch=478 (Abruf am 31. Januar 2010).

27 Vgl. Kopenhagener Dokument, a.a.O (Fn. 25), S. 9.

28 Vgl. Kurt P. Tudyka, a.a.O. (Fn. 23), S. $103-107$.

29 Vgl. OSCE / ODIHR, Election Observation Handbook, Warschau 2007, S. 25, http://www. osce.org/publications/odihr/2005/04/14004_240_en.pdf (Abruf am 20. Januar 2010).

30 Vgl. OSCE / ODIHR, a.a.O. (Fn. 26), S. 2. 
cken oder Widersprüchlichkeiten im Wahlgesetz, verzerrt oder ungleichgewichtig zugeschnittenen Wahlkreisen und Unregelmäßigkeiten in den Wählerverzeichnissen, reichen über eine unausgewogene Berichterstattung nationaler Medien und die missbräuchliche Verwendung staatlicher Ressourcen zugunsten einer Partei oder eines Kandidaten im Vorfeld des Urnenganges bis hin zu Einschüchterungen der Wähler am Wahltag sowie Unregelmäßigkeiten bei der Stimmabgabe. Ferner sind mögliche Regelverstöße bei der Auszählung der Stimmen in einzelnen Wahllokalen sowie beim Zusammenzählen der lokalen Ergebnisse auf regionaler wie auf nationaler Ebene sowie Unregelmäßigkeiten im gerichtlichen oder außergerichtlichen Verfahren zur Beilegung von Streitigkeiten in Betracht zu ziehen. ${ }^{31}$

Mit dem komplexen Prozessverständnis geht die Einsicht einher, die Bewertung einer Wahl anhand der Eigenschaften „frei“ und „fair" keinen allgemeingültigen Kriterien unterwerfen zu können, sondern unter angemessener Würdigung spezifischer Umstände in den jeweiligen Gesamtzusammenhang einzubetten. Sicherlich kann nicht jeder Urnengang beispielsweise in Transformationsländern alle internationalen Standards auf Anhieb erfüllen. Entscheidend ist bei der Bewertung der demokratischen Qualität einer Wahl deshalb die Frage, inwiefern Unregelmäßigkeiten und Ungenauigkeiten systematischer Natur sind und einem Muster entsprechen, das den Wählerwillen in seinem Gesamtergebnis verfälschen und somit die Rechtmäßigkeit des Urnengangs gefährden könnte. ${ }^{32}$

Die EU hat in ihren Vertragswerken ebenfalls klare Bekenntnisse zum Schutze der Menschenrechte und Grundfreiheiten sowie zu Demokratie und Rechtsstaatlichkeit verankert, auf deren Durchsetzung sie auch im Bereich der Entwicklungszusammenarbeit hinwirkt. In einer Kommissionsmitteilung vom 11. April 2000 über „Wahlunterstützung und Wahlbeobachtung durch die EU“ wird die Wichtigkeit von Wahlen als „wesentlicher Schritt im Demokratisierungsprozeß“ im Hinblick auf die Menschenrechte aus zwei Gründen hervorgehoben: „Zum einen können die Menschen dabei ihrem politischen Willen Ausdruck verleihen. Zum anderen müssen Wahlen, um wirklich frei und fair zu sein, in einem Klima stattfinden, in dem die Menschenrechte gewahrt werden. "33 Freien und fairen Wahlen wird eine entscheidende Rolle bei der Beilegung interner Konflikte sowie der Legitimation staatlicher Autorität zugewiesen. ${ }^{34}$ Entsprechend sei oberstes Ziel des Instruments internationaler Wahlbeobachtung, „die Demokratie in den jeweiligen Ländern fest zu verankern und durch Förderung des entsprechenden Know-hows in diesen Ländern sich selber überflüssig zu machen “35. In ihren Bemühungen konzentriert sich die EU hierbei auf Länder, die nicht

31 Vgl. Ekkehard Münzing, a.a.O. (Fn. 5), S. 96 - 112; Peter Eicher, a.a.O. (Fn. 8), S. 269; Christian Strohal, Democratic Elections and their Monitoring: Can this OSCE Success Story Be Sustained?, in: Wolfgang Benedek / Wolfram Karl / Anja Mihr / Manfred Nowak (Hrsg.), European Yearbook on Human Rights, Wien u.a. 2009, S. 247 - 264, S. 252, S. 260 - 262; Andreas Schedler, Elections Without Democracy. The Menue of Manipulation, in: Journal of Democracy, 13. Jg. (2002), H. 2, S. $36-50$.

32 Vgl. Kurt P. Tudyka, a.a.O. (Fn. 23), S. 232.

33 Kommission der Europäischen Gemeinschaften, Mitteilung der Kommission über Wahlunterstützung und Wahlbeobachtung durch die EU, Brüssel 2000, S. 5, http://eur-lex.europa.eu/LexUriServ/LexUriServ.do?uri=COM:2000:0191:FIN:DE:PDF (Abruf am 21. August 2010).

34 Vgl. Kommission der Europäischen Gemeinschaften, Arbeitsdokument der Kommissionsdienststellen zur Umsetzung der Mitteilung über Wahlunterstützung und Wahlbeobachtung, Brüssel 2003, S. 3, http://ec.europa.eu/external_relations/human_rights/election_observation/docs/sec _2003_1472_de.pdf (Abruf am 21. August 2009).

35 Kommission der Europäischen Gemeinschaften, a.a.O. (Fn. 33), S. 6. 
zu den Teilnehmerstaaten der OSZE gehören, insbesondere in Afrika, Lateinamerika, Asien und im Nahen Osten. ${ }^{36}$

Am 27. Oktober 2005 haben 23 Organisationen und Institutionen die Declaration of Principles for International Election Observation sowie den Code of Conduct for International Election Observers ${ }^{37}$ verabschiedet. Anknüpfend an bereits bestehende Prinzipien und Verhaltenskodizes der beteiligten Einrichtungen ${ }^{38}$ werden darin die Grundsätze demokratischer Wahlen und ihrer Beobachtung auf eine gemeinsame Basis gestellt. Internationale Wahlbeobachtung wird hierbei definiert als

„the systematic, comprehensive and accurate gathering of information concerning the laws, processes and institutions related to the conduct of elections and other factors concerning the overall electoral environment; the impartial and professional analysis of such information; and the drawing of conclusions about the character of electoral processes based on the highest standards for accuracy of information and impartiality of analysis“ 39 .

\section{Die Beobachtung von Wablen als strukturierter und standardisierter Prozess: Missionen der OSZE und der EU}

Internationale Wahlbeobachtungsmissionen sind nicht notwendigerweise darauf ausgerichtet, den Wahlablauf am Tag des Urnenganges in allen Wahllokalen zu verfolgen. Das Ziel einer Mission besteht allerdings auch nicht darin, die beobachtete Wahl formal zu zertifizieren, sondern sie an den internationalen Standards einer freien und fairen Wahl sowie an den nationalen Vorgaben zu messen. Um die Einhaltung dieser Kriterien anhand der beobachteten Ereignisse qualifiziert bewerten zu können, wurden zwischenzeitlich standardisierte Verfahren etabliert. Hierbei fiel der OSZE eine Vorreiterrolle zu, und zahlreiche Elemente der ursprünglich von ODIHR entwickelten Methodologie zur Beobachtung und Bewertung von Wahlen wurden zwischenzeitlich von anderen Organisationen übernommen, darunter auch die EU. ${ }^{40}$

Die ODIHR-Methodologie, die im Election Observation Handbook ${ }^{41}$ zusammengefasst ist, geht erheblich über die Beobachtung der Ereignisse am Wahltag hinaus und voll-

36 Vgl. European Commission, Handbook for European Union Election Observation, Brüssel 2008, http://ec.europa.eu/europeaid/what/human-rights/election_observation_missions/documents/eu_election_observation_handbook_en.pdf, S. 7 (Abruf 30. Januar 2010).

37 Declaration of Principles for International Election Observation and Code of Conduct for International Election Observers, New York 2005, http://www.idea.int/publications/other/upload/ dec_obs_coc.pdf (Abruf am 28. Oktober 2009).

38 Der Verhaltenskodex der OSZE kann beispielsweise dem Election Observation Handbook entnommen werden; vgl. OSCE / ODIHR, a.a.O. (Fn. 29), S. 22. Im Handbook for European Union Election Observation finden sich die allgemeinen Prinzipien für EU-Missionen und die Verhaltensregeln der EU-Beobachter; vgl. European Commission 2008, a.a.O. (Fn. 36), S. 23 26, S. $10 \mathrm{f}$.

39 Declaration of Principles for International Election Observation, a.a.O. (Fn. 37), S. 2.

40 Vgl. Peter Eicher, a.a.O. (Fn. 8), S. 267; Christian Strohal, a.a.O. (Fn. 31), S. 248; Victor-Yves Ghebali, a.a.O. (Fn. 17), S. 251; Peter van Ham, EU-OSCE Relations. Partners or Rivals in Security, in: Knud Erik Jorgensen (Hrsg.), The European Union and International Organizations, London / New York 2009, S. 131 - 148, S. 141; European Commission, a.a.O. (Fn. 36), S. 210.

41 Vgl. OSCE / ODIHR, a.a.O. (Fn. 29). 
zieht sich auf vier Ebenen ${ }^{42}$ : Zunächst ergründet eine Bedarfsermittlungsmission die rechtlichen, organisatorischen und politischen Rahmenbedingungen des Wahlganges und untersucht, ob und in welchem Ausmaß die Entsendung einer Beobachtermission empfehlenswert ist. In einem zweiten Schritt erfolgt der Einsatz eines Kernteams, das bereits Wochen oder Monate vor dem Wahltermin seine Arbeit in dem jeweiligen Land aufnimmt. Das Kernteam führt die von der Bedarfsermittlungsmission begonnene Analyse fort und setzt sich zudem mit weiteren Aspekten auseinander, etwa dem Fortgang der Registrierung von Parteien, Kandidaten und Wahlberechtigten, dem Verlauf des Wahlkampfes sowie der Berichterstattung in den Medien im Vorfeld der Wahl, aber auch mit der Durchführung des Wahlprozesses und der Auszählung der Stimmen am Wahltag sowie der Lösung von Konflikten im Anschluss an den Wahlgang. Die Arbeit des Kernteams wird durch den Einsatz von Langzeit-Wahlbeobachtern als dritte Ebene ergänzt, die das Geschehen in den einzelnen Regionen des Landes verfolgen und die logistischen Voraussetzungen für den etwa ein- bis anderthalbwöchigen Einsatz von Kurzzeit-Wahlbeobachtern als vierte Ebene einer Mission schaffen. Deren Anzahl kann bis zu mehrere hundert Personen betragen, wodurch systematische Beobachtungen des Wahlgeschehens in individuellen Wahllokalen am Wahltag, aber auch im unmittelbaren Zeitraum davor und danach ermöglicht werden. Diese werden vornehmlich auf standardisierte Weise erfasst und aufbereitet, um die Vergleichbarkeit der individuellen Beobachtungen sicherzustellen.

Verstärkt werden können die Beobachtungsmissionen von ODIHR durch Delegationen der Parlamentarischen Versammlungen der OSZE, der NATO und des Europarats, die im Rahmen von „konsolidierten Beobachtungen“ unter einem gemeinsamen Dach auf dieselben standardisierten Formulare zurückgreifen und deren Ergebnisse in einen gemeinsamen Bericht der beteiligten Institutionen einfließen, um das Risiko widersprüchlicher Bewertungen oder Schlussfolgerungen verschiedener internationaler Einrichtungen möglichst auszuschließen. ${ }^{43}$

Peter Eicher macht allerdings darauf aufmerksam, dass mögliche politische Erwägungen parlamentarischer Delegationen bei der Bewertung eines Urnenganges zu Konflikten mit ODIHR führen können:

„Parliamentary observers and the ODIHR can usually cooperate constructively, but in some instances the politics of parliamentary observation can distort an observation mission's findings. This has, at times, led to assignments being softened. I recall one parliamentary delegation leader telling me after a particularly hard-fought and unsatisfactory negotiation of a preliminary election statement: 'The problem, Peter, is that you wanted to tell the truth."'44

Neben Beobachtungsmissionen, die aus allen der vier dargestellten Komponenten bestehen, hat ODIHR zwischenzeitlich seine Methodologie für spezielle Erfordernisse modifiziert. So sind nun auch Missionen ohne Kurzzeit-Wahlbeobachter, die Entsendung von Expertenteams und Wahlbewertungsmissionen (election assessment missions) denkbar. Letztere kom-

42 Vgl. für die Ausführungen dieses Absatzes Rick Fawn, a.a.O. (Fn. 9), S. 1135 f.; OSCE / ODIHR, a.a.O. (Fn. 29), S. 23 - 33.

$43 \mathrm{Vgl}$. Bruno Haller, Election Observation by the Parliamentary Assembly of the Council of Europe (PACE), in: Human Rights Law Journal, 26. Jg. (2005), H. 5 - 8, S. 160 - 166; Kurt P. Tudyka, a.a.O. (Fn. 23), S. 231 f.; OSCE / ODIHR, a.a.O. (Fn. 29), S. 75 f.; Rick Fawn, a.a.O. (Fn. 9), S. 1136.

44 Peter Eicher, a.a.O. (Fn. 8), S. 270. 
men seit dem Jahr 2002 insbesondere in langjährigen oder etablierten Demokratien zum Einsatz und konzentrieren sich auf spezielle Fragestellungen oder die Umsetzung bewährter Methoden, ohne jedoch die Geschehnisse am Wahltag in der Fläche zu beobachten. ${ }^{45}$

Die Modifizierung der ODIHR-Methodologie und die Ausweitung der Beobachtungsaktivitäten auf alle OSZE-Teilnehmerstaaten kann nicht nur als Reaktion auf die manifesten Unregelmäßigkeiten bei den US-amerikanischen Präsidentschaftswahlen im November $2000^{46}$ verstanden werden, sondern auch als Ergebnis von Spannungen zwischen den OSZE-Teilnehmerstaaten. Wie bereits zuvor ausgeführt, haben die Berichte von OSZEWahlbeobachtungsmissionen mit Belegen für umfangreiche Wahlfälschungen entscheidende Auswirkungen auf politische Veränderungsprozesse in ehemaligen Teilrepubliken der Sowjetunion gehabt, insbesondere im Vorfeld der „farbigen Revolutionen“ in Georgien, in der Ukraine und in Kirgisien. Bereits im Juli 2004 und somit noch vor den Ereignissen in der Ukraine und in Kirgisien verabschiedeten die Staatspräsidenten von insgesamt neun ehemals sowjetischen Teilrepubliken unter russischer Führung eine Erklärung, die die Aktivitäten der OSZE als Verletzung nationaler Souveränitätsrechte interpretierte und der OSZE die Verwendung „doppelter Standard““ unterstellte, da sie einigen ihrer Teilnehmerstaaten sowie spezifischen Fragestellungen eine zunehmend selektive Aufmerksamkeit zukommen lasse, ohne jedoch die spezifischen Gegebenheiten und Besonderheiten einzelner Staaten genügend zu berücksichtigen. Auch wurde den Wahlbeobachtungsmissionen von ODIHR mangelnde Objektivität unterstellt: Solange die in den Augen westlicher Regierungen „richtige Seite“ gewinne, würden Beobachter der OSZE Unregelmäßigkeiten gerne übersehen, so das damalige Argument. ${ }^{47}$ In der russischen Zeitschrift „International Affairs" fasste der russische Diplomat Vladimir Gorovoi die Kritik seines Landes an den Missionen von ODIHR in folgende Worte:

\begin{abstract}
„Unfortunately, the activity of international observers is being increasingly used both by opposition forces within a particular state and by foreign, champions of democracy' as a tool for exerting political pressure within that country. As a result, by manipulating facts, spin doctors and certain politicians stoke internal political tensions during and after elections. The events in Ukraine in 2004 and in Kyrgyzstan in 2005 showed a great diversity of means and methods of destabilizing the situation. In all instances, such actions were triggered by elections, or rather, their results. The findings and conclusions made by Western observers were also an important factor, especially when violations were in some cases regarded as insubstantial, while in other cases the same breaches were used as grounds to report deviations from the fundamental principles of democracy [...]. Unfortunately, very much depends not on comments and evaluations by rank and file observers as on coordinating structures that use isolated facts to build a complete picture and make final conclusions. [...] The danger is that the institution, which was originally set up as an independent and democratic instrument of ensuring the openness and transparency of the electoral process, could discredit itself. " 48
\end{abstract}

45 Vgl. OSCE / ODIHR, a.a.O. (Fn. 29), S. 14; Peter Eicher, a.a.O. (Fn. 8), S. 267; Rachel Ricker, a.a.O. (Fn. 7), S. 1382.

46 Vgl. Eric C. Bjornlund, a.a.O. (Fn. 7), S. 3-6.

47 Vgl. Rick Fawn, a.a.O. (Fn. 9), S. 1141 f.; Susan Stewart, Russland und die OSZE. Zum Spannungsverhältnis zwischen Gleichheit und Verpflichtung, SWP-Aktuell, Nr. 66, Berlin 2007, http:// www.swp-berlin.org/common/get_document.php?asset_id=4573 (Abruf am 21. Februar 2010).

48 Vladimir Gorovoi, CIS Election Observer Missions in Commonwealth States, in: International Affairs. A Russian Journal of World Politics, Diplomacy and International Relations, 52. Jg. (2005), H. 2, S. $79-87$, S. 83 f. 
Russland forderte vor diesem Hintergrund institutionelle Reformen innerhalb der OSZE, die die Autonomie von ODIHR spürbar begrenzt und die abschließende Bewertung von Wahlen politischen Gremien zugeordnet hätte. ${ }^{49}$ Eine unabhängige Beobachtung von Wahlen durch ODIHR wäre damit faktisch unmöglich geworden. Zudem kamen zunehmend eigene Wahlbeobachtungsmissionen der GUS zum Einsatz, deren Bewertungen beispielsweise beim ersten und zweiten Wahlgang sowie bei der Wiederholung des zweiten Wahlganges der ukrainischen Präsidentschaftswahlen im Oktober, November und Dezember 2004 jeweils konträr zu den Schlussfolgerungen der OSZE lagen. ${ }^{50}$ Darüber hinaus legte Russland der geplanten ODIHR-Mission zur Beobachtung der Parlamentswahlen am 2. Dezember 2007 derartige Restriktionen auf, dass ODIHR sich nicht in der Lage sah, eine Beobachtungsmission entsprechend seiner Methodologie durchzuführen, und die formell in Übereinstimmung mit dem Kopenhagener Dokument ergangene - Einladung der russischen Behörden absagte. Dies wiederholte sich im Vorfeld der russischen Präsidentschaftswahlen am 2. März 2008. ${ }^{51}$ Beobachter bewerteten dieses Vorgehen als Präzedenzfall, der anderen Staaten einen möglichen Weg zur faktischen Umgehung der verpflichtenden Einladung von ODIHR-Wahlbeobachtern aufzeigte. ${ }^{52}$

Deutlich wurde spätestens durch diese Entwicklung, dass die russische Kritik an den OSZE-Kriterien freier und fairer Wahlen sowie an der Objektivität des ODIHR nur vordergründig war und es in Wirklichkeit um tiefer gelagerte politische Interessen ging. Um eine Schwächung des Instruments der Wahlbeobachtung zu vermeiden, legte ODIHR jedoch fortan zunehmend Wert auf eine stärkere personelle Ausgewogenheit unter den OSZE-Staaten bei der Rekrutierung des Personals für Beobachtermissionen sowie auf die Besetzung auch sehr hochrangiger Posten innerhalb seiner Missionen durch Vertreter aus ehemaligen Teilrepubliken der Sowjetunion. Zudem erweiterte die OSZE die Wahlbeobachtungsaktivitäten des ODIHR auf die Gesamtheit ihrer Teilnehmerstaaten und adressiert seitdem ihre Bemühungen verstärkt auch auf etablierte Demokratien, zumeist in Form von Experten- und Wahlbewertungsmissionen. ${ }^{53}$

\section{Die Beobachtung der Bundestagswahl 2009 durch die OSZE}

Anlässlich der Bundestagswahl 2009 richtete ODIHR seine Blicke vor diesem Hintergrund erstmals auf eine Abstimmung in Deutschland. Zunächst bereiste eine dreiköpfige Bedarfsermittlungsmission im Sommer 2009 die Bundesrepublik. Ihr folgte eine 15-köpfige Wahlbewertungsmission für einen Zeitraum von etwa zweieinhalb Wochen vor, während und nach dem Wahltag am 27. September 2009.

49 Vgl. Christian Strohal, a.a.O. (Fn. 31), S. 256; Susan Stewart, a.a.O. (Fn. 47).

50 Vgl. Victor-Yves Ghebali, a.a.O. (Fn. 17), S. 254; Rick Fawn, a.a.O. (Fn. 9), S. $1141-1145$.

51 Vgl. Christian Strohal, a.a.O. (Fn. 31), S. 262; Solveig Richter / Wolfgang Zellner, Ein neues Helsinki für die OSZE? Chancen für eine Wiederbelebung des europäischen Sicherheitsdialogs, SWP-Aktuell, Nr. 81, Berlin 2008, http://www.swp-berlin.org/common/get_document. php?asset_id=5489 (Abruf am 21. Februar 2010).

52 Vgl. Judith Kelley, a.a.O. (Fn. 10), S. 769.

53 Vgl. Rick Fawn, a.a.O. (Fn. 9), S. 1145 - 1153; Victor-Yves Ghebali, a.a.O. (Fn. 17), S. 253 - 255. 
Der im August 2009 veröffentlichte Bericht ${ }^{54}$ der Bedarfsermittlungsmission des ODIHR, die Mitte Juli 2009 zahlreiche Gespräche mit Vertretern staatlicher Stellen, der Medien und der im Bundestag vertretenen Parteien geführt hatte, betonte das hohe Maß an öffentlichem Vertrauen in die Integrität des Wahlprozesses. Die rechtlichen Rahmenbedingungen böten eine fundierte Basis für die Durchführung demokratischer Wahlen, und die sich über vier Ebenen erstreckende Wahlbehörde genösse ein überwältigendes Maß an Vertrauen. Zudem agierten die Parteien unter gleichen Wettbewerbsbedingungen und hätten gleichmäßigen Zugang zu den öffentlichen wie privaten Medien, die die Wähler mit mannigfaltigen und ausgewogenen Informationen über die zur Wahl stehenden Kandidaten versorgten. Kritisch angemerkt wurde allerdings, dass die Wahlgesetzgebung keine expliziten Festlegungen für die Anwesenheit von Wahlbeobachtern vorsähe und deshalb nicht vollständig im Einklang mit den Bestimmungen von $\$ 8$ des Kopenhagener Dokuments stünde, wenngleich der öffentliche Charakter des Wahlprozesses in zahlreichen Einzelnormen der gesetzlichen Wahlbestimmungen bekräftigt werde und die Beobachtung der Wahlen an keiner Stelle des Gesetzes ausgeschlossen würde. Aufgeführt wurde zudem, dass die Finanzierung der Wahlkampfaktivitäten nicht in der Wahlgesetzgebung geregelt sei, es weder eine Deckelung der Wahlkampfausgaben noch eine Beschränkung einzelner Bezugsquellen gäbe und Kandidaten die Herkunft der Mittel zur Finanzierung ihres Wahlkampfes nicht offenzulegen hätten.

Die Tatsache, dass das Bundesverfassungsgericht Teile des Wahlrechts in seinem Urteil vom 3. Juli $2008^{55}$ als verfassungswidrig eingestuft hat, da das so genannte negative Stimmgewicht gegen die Grundsätze der Gleichheit und der Unmittelbarkeit der Wahl verstoße, und das Gericht dem Gesetzgeber eine Frist bis zum 30. Juni 2011 zur Behebung dieses verfassungswidrigen Zustands gesetzt hat, wurde in dem Bedarfsermittlungsbericht allerdings nur am Rande erwähnt - obgleich im Vorfeld der Wahl in Deutschland eine öffentliche Debatte ${ }^{56}$ hinsichtlich der Frage geführt wurde, ob die Wahl eines neuen Bundestages nach nunmehr offiziell als verfassungswidrig erklärtem Wahlrecht statthaft sei, zumal die Zeitspanne zwischen dem Urteil des Verfassungsgerichts im Juli 2008 und dem Wahltag am 27. September 2009 bei bestehendem politischen Konsens in dieser Frage vielfach als ausreichend für eine Modifizierung des Wahlgesetzes und damit eine Heilung des verfassungswidrigen Zustands angesehen wurde. Verstärkte Resonanz erhielt die Debatte zudem dadurch, dass es im Vorfeld des Urnenganges als nicht ausgeschlossen galt, dass sich eine

54 OSCE / ODIHR, a.a.O. (Fn. 4). Soweit nicht anderweitig angemerkt, sind alle Angaben dieses und des folgenden Absatzes diesem Bericht der ODIHR-Bedarfsermittlungsmission entnommen.

55 Vgl. Daniel Lübbert, Negative Stimmgewichte und die Reform des Bundestags-Wahlrechts, Deutscher Bundestag, Wissenschaftliche Dienste, Info-Brief, WD 8-3000-020/09, http://www.bundestag.de/dokumente/analysen/2009/negative_stimmgewichte.pdf (Abruf am 8. Januar 2010). Vgl. auch Dieter Nohlen, Erfolgswertgleichheit als fixe Idee oder: Zurück zu Weimar? Zum Urteil des Bundesverfassungsgerichts über das Bundeswahlgesetz vom 3. Juli 2008, in: ZParl, 40. Jg. (2009), H. 1, S. 179 - 195; Joachim Behnke, Überhangmandate bei der Bundestagswahl 2009. Eine Schätzung mit Simulationen, in: ZParl, 40. Jg. (2009), H. 3, S. 620 - 636; Eric Linhart, Mögliche Auswirkungen von Grabenwahlsystemen in der Bundesrepublik Deutschland. Theoretische Überlegungen und Simulationen, in: ZParl, 40. Jg. (2009), H. 3, S. 637 - 660.

56 Vgl. etwa Heribert Prantl, Aus Fünf und Sechs mach Sieben und Acht - so ist's vollbracht. Hexeneinmaleins und Wahlrecht: Warum Karlsruhe es erlaubt, dass der Bundestag im September auf verfassungswidrige Weise gewählt wird, in: SZ vom 30. Juni 2009, S. 5. 
Regierung auf eine ausschließlich durch Überhangmandate entstehende Parlamentsmehrheit würde stützen können. ${ }^{57}$

Basierend auf ihren Erkenntnissen empfahl die Bedarfsermittlungsmission die Entsendung einer Wahlbewertungsmission. Diese sollte keine umfassende und systematische Beobachtung der Geschehnisse am Wahltag beinhalten, jedoch den Urnengang in einzelnen Bundesländern verfolgen und sich in ihrer Bewertung des Geschehens insbesondere auf die gesetzlichen Rahmenbedingungen und die Administration des Wahlganges sowie Fragen der Wahlkampffinanzierung, der Briefwahl und des Zugangs für Wahlbeobachter konzentrieren.

Anknüpfend an den Bericht der Bedarfsermittlungsmission wurde zwischen dem 13. September und dem 1. Oktober 2009 eine solche Wahlbewertungsmission nach Deutschland entsandt, die 15 Experten aus 13 OSZE-Teilnehmerstaaten umfasste und am 14. Dezember 2009 ihren Abschlussbericht vorlegte. Dieser bewertete die Durchführung der Wahlen in ihrer Gesamtheit als äußerst positiv:

„The 2009 federal parliamentary elections confirmed a solid experience in conducting democratic elections in Germany. They demonstrated an open, pluralistic and competitive process, founded on the respect for fundamental freedoms, equitable conditions for all contestants, the efficiency and professionalism of the election administration as well as a high level of public confidence in the overall integrity of the electoral process." 58

Bestätigt wurde zudem die Einschätzung der vorangegangenen Bedarfsermittlungsmission, wonach die rechtlichen Rahmenbedingungen eine insgesamt fundierte Basis für die Durchführung demokratischer Wahlen böten. Dennoch wurde eine Überarbeitung des Wahlgesetzes insbesondere hinsichtlich dreier Aspekte empfohlen:

(1) Die Entscheidungen der Wahlbehörden sollten im Vorfeld des Wahltages gerichtlich überprüft werden können. Entscheidungen der Landes- und der Kreiswahlausschüsse könnten zwar innerhalb einer Frist von drei Tagen vor dem Bundeswahlausschuss angefochten werden, doch könnten dessen Entscheidungen vor dem Urnengang nicht auf dem Rechtsweg hinterfragt werden. Auch sollten Entscheidungen des Wahlprüfungsausschusses des Deutschen Bundestages, der laut Grundgesetz für die Wahlprüfung zuständig ist, zu Einsprüchen im Anschluss an den Wahltag auch von einzelnen Wahlberechtigten vor dem Bundesverfassungsgericht angefochten werden können, ohne dass - wie bislang - die $\mathrm{Zu}-$ lässigkeit eines Einspruchs formell an die schriftliche Unterstützung durch einhundert weitere Wahlberechtigte geknüpft wird.

(2) Die Zulassung von Parteien, Wahllisten und einzelnen Kandidaten zur Wahl sollte anhand von präzisen, objektiven und messbaren Kriterien erfolgen, um dem möglichen Eindruck subjektiver Entscheidungen entgegenzuwirken. Damit griffen die ODIHR-Experten die auch in der Öffentlichkeit kritisierten Entscheidungen des Bundeswahlausschusses vom 6. August 2009 auf, der mehreren Kleinparteien die Zulassung zur Bundestagswahl versagt hatte. Des Weiteren kritisierte der Bericht, dass dem Bundeswahlausschuss vor-

57 Vgl. Joachim Behnke, a.a.O. (Fn. 5).

58 Vgl. OSCE / ODIHR, Federal Republic of Germany. Elections to the Federal Parliament (Bundestag), OSCE / ODIHR Election Assessment Mission Report, Warschau 2009, S. 1, http:// www.osce.org/documents/odihr-el/2009/12/42097_en.pdf (Abruf am 5. Januar 2009). Soweit nicht anderweitig angemerkt, sind die im Folgenden dargestellten Ergebnisse der ODIHR-Wahlbewertungsmission diesem Bericht entnommen. 
nehmlich Vertreter etablierter Parteien angehörten, was insbesondere bei Fragen der Zulassung einzelner Parteien die Gefahr von Interessenkonflikten mit sich bringe.

(3) Zudem sollten in der Wahlgesetzgebung Vorkehrungen getroffen werden, die die Anwesenheit von unparteiischen nationalen wie internationalen Wahlbeobachtern gestatten und diesen ausdrücklich Zugang zu allen Stufen des Wahlgeschehens einräumen. Die öffentliche Natur des Wahlverfahrens sei zwar gesetzlich festgeschrieben und die Beobachter der OSZE hätten vor, während und nach der beobachteten Wahl vollständige Einsicht in das Wahlgeschehen nehmen können, doch sei eine ausdrückliche gesetzliche Festlegung hinsichtlich der Zulässigkeit von Wahlbeobachtern Bestandteil der zwischen den OSZEStaaten vereinbarten Maßgaben. Um diesen vollständig zu entsprechen und denkbare Unsicherheiten auszuräumen, sollte die Gesetzgebung entsprechend ergänzt werden.

Zusätzlich zu diesen drei Hauptaspekten unterbreitete der OSZE-Bericht einige konkrete Vorschläge zur Überarbeitung und Ergänzung von Detailregelungen des Wahlprozesses, insbesondere zu folgenden Punkten: das Ausmaß der zu tolerierenden Größenabweichungen bei der Festlegung von Wahlkreisgrenzen; die Umstände zu verschiebender Abstimmungen innerhalb eines Wahlkreises in Folge des Todes eines Kandidaten; Verlängerung der Einspruchsfrist zur Verifizierung von Eintragungen in das Wählerverzeichnis; verbesserte Transparenz der Parteienfinanzierung durch eine schnellere öffentliche Bekanntmachung höherer Spendensummen ${ }^{59}$ an Parteien sowie eine raschere Veröffentlichung der jährlichen Rechenschaftsberichte der Parteien; Offenlegung der Kriterien der großen Radio- und Fernsehanstalten bei der Verteilung von Zeitfenstern zur Ausstrahlung von Wahlwerbespots auf die einzelnen Parteien; genauere Dokumentation der in einzelnen Wahllokalen empfangenen, verwendeten und unbenutzten Stimmzettel; verbesserte Prävention möglicher Gesetzesverstöße bei der Briefwahl.

\section{Fazit: Konsequenzen für die Erarbeitung der Wahlgesetzgebung}

Die internationale Beobachtung von Wahlen ist ein wichtiges Instrument der Demokratisierungshilfe, das insbesondere seit den 1990er Jahren systematisch weiterentwickelt und gestärkt wurde. Zahlreiche internationale sowie nichtstaatliche Organisationen führen mittlerweile Beobachtungsmissionen durch, darunter mit deutscher Beteiligung die OSZE und die EU. Ausgehend von dem Standard „freier und fairer Wahlen“ berücksichtigt die durch ODIHR entwickelte Methodologie bei der Durchführung von Beobachtungsmissionen, an der sich zahlreiche Organisationen orientieren, die Gesamtheit aller einem Urnengang zugrunde liegenden politischen, rechtlichen, administrativen und öffentlichen Rahmenbedingungen und legt der Bewertung des Gesehenen ein komplexes Verständnis des Wahlprozesses zugrunde.

59 Die Empfehlung einer rascheren Veröffentlichung von Großspenden wurde mit einem Beschluss des Ältestenrats des Deutschen Bundestages vom 28. Januar 2010 bereits umgesetzt. Ausschlaggebend hierfür war jedoch weniger der OSZE / ODIHR-Bericht, sondern vielmehr die öffentliche Debatte im Nachgang zu einer bekannt gewordenen Millionenspende an eine der beiden Regierungsparteien. Vgl. Zeit Online, Parteienfinanzierung: Großspenden sollen sofort publik werden, 28. Januar 2010, http://www.zeit.de/politik/deutschland/2010-01/partei-spenden-oeffentlich (Abruf am 1. Februar 2010). 
Auch zur Entkräftung des zwischenzeitlich ergangenen Vorwurfs doppelter Standards bei der Bewertung von Wahlen ihrer Teilnehmerstaaten konzentrieren sich die Beobachtungsaktivitäten der OSZE seit einigen Jahren nicht mehr ausschließlich auf die Länder des ehemaligen Warschauer Paktes, die sich in demokratischen Transformationsprozessen befinden, sondern wenden sich zunehmend auch den Wahlgängen in den etablierten Demokratien unter ihren Teilnehmerstaaten zu. Entsprechend erfolgte die Beobachtung der Wahl zum 17. Deutschen Bundestag am 27. September 2009 durch ODIHR nicht vor dem Hintergrund von Zweifeln an der Integrität des Prozesses, wie dies in der medialen Berichterstattung partiell suggeriert wurde, sondern im Einklang mit dieser Weiterentwicklung der bewährten OSZE-Praxis. In Übereinstimmung mit dieser Praxis handelte es sich bei der nach Deutschland entsandten Mission nicht um eine Wahlbeobachtung im klassischen Sinne, zu deren Aufgaben auch die umfassende und systematische Beobachtung der Geschehnisse am Wahltag gehört hätte, sondern um eine Wahlbewertungsmission, deren 15-köpfiges Expertenteam sich insbesondere mit den Rahmenbedingungen des Urnenganges auseinandersetzte.

Selbst wenn die Gesamtbewertung dieser Rahmenbedingungen äußerst positiv ausgefallen ist, benennt der Abschlussbericht der OSZE-Wahlbewertungsmission einzelne rechtliche Festlegungen, die aus internationaler Sicht verbesserungswürdig erscheinen. Die von der OSZE unterbreiteten Vorschläge könnten Eingang in die Überarbeitung der Wahlgesetzgebung finden, die das Bundesverfassungsgericht dem Gesetzgeber bis zum 20. Juni 2011 aufgegeben hat. Sollte sich der Bundestag dazu entschließen, die Hinweise der ODIHR-Experten bei der Novellierung des Bundeswahlgesetzes zu berücksichtigen, würde dies international sicherlich nicht nur als ein deutliches Bekenntnis zum OSZE-System der kollektiven Sicherheit und seiner Bedeutung verstanden werden, sondern der Reputation der OSZE und des ODIHR sowie deren Empfehlungen gerade auch unter anderen Teilnehmerstaaten stärkeren Nachdruck verleihen.

\title{
Die Bundestagswahl 2009 im Spiegel der repräsentativen Wahlstatistik
}

\author{
Eckhard Jesse
}

Die SPD erreichte bei der Bundestagswahl 2009 in der Gruppe der 25- bis 34-jährigen Männer im Osten Deutschlands bloß 12,8 Prozent, lag damit nicht nur hinter der CDU (25,0 Prozent) und der Linken (19,7 Prozent), sondern auch hinter der FDP (17,8 Prozent); und bei den 18- bis 24-Jährigen - gleiches Geschlecht, gleiche Region - schnitten die „übrigen Parteien“ (20,8 Prozent), darunter die Piratenpartei ${ }^{1}$ und die NPD (8,5 Prozent),

1 Das Statistische Bundesamt hatte die „Piraten“ zunächst nicht berücksichtigt, sie später jedoch einbeziehen wollen. Dies war nur für sieben Länder möglich. Wie die Angaben für Brandenburg (14,0 Prozent), Berlin-Ost (16,4 Prozent) und Mecklenburg-Vorpommern (14,2 Prozent) verdeutlichen, kam die Partei bei den jungen Männern im Osten auf bessere Ergebnisse als die NPD. Wären die „Piraten“ auch in Sachsen angetreten, dann hätten die „übrigen Parteien“ in dieser Kategorie sogar an der ersten Stelle gelegen. 